\author{
MAGDALENA LIPIŃSKA
}

Université de Łódź

\title{
LES FONCTIONS DU LANGAGE ET LA DÉIXIS DANS LES SLOGANS PUBLICITAIRES FRANÇAIS
}

\begin{abstract}
Lipińska Magdalena, Les fonctions du langage et la déixis dans les slogans publicitaires français [Speech functions and deixis in French advertising slogans]. Studia Romanica Posnaniensia, Adam Mickiewicz University Press, Poznań, vol. XXXVI: 2009, pp. 97-109. ISBN 978-83-232-2035-0. ISSN 0137-2475.
\end{abstract}

The article describes speech functions and deixis in French advertising slogans. Such functions as communicative, phatic, and imperative are treated as the defining ones, whereas the last one is usually consciously hidden. The poetic and the expressive functions are considered important yet not the necessary ones, while the denotative one is often blurred. The metalinguistic function is considered secondary, however it cannot be excluded. The type of message imposes deixis' referents and syntactic functions of the latter, e.g. the subject determines the brand, producer or the service donor, a potential customer or a person who used a given product and is satisfied with it. The slogan's deixis focuses around the major elements of the product sale.

\section{INTRODUCTION}

Parce que je le vaux bien (L'Oréal); Qui sème les fleurs, récolte la tendresse (Interflora). Ce qui saute aux yeux dans ces phrases, c'est le grand rôle de la déixis, dans la première, et dans la seconde, le caractère autonyme et en même temps l'importance des fonctions métalinguistique et poétique. Quel est le statut des fonctions du langage dans les slogans publicitaires français ? Quels sont les traits caractéristiques des déictiques dans ces énoncés ? Le présent travail se propose de répondre à ces questions. Après avoir esquissé l'état des recherches qui concernent les slogans, on présentera les traits définitoires de ces formes autonymes. On prendra en considération les fonctions du langage définies par $R$. Jakobson en essayant de préciser leur spécificité dans les énoncés précités. Nous analyserons également les types de référents des déictiques et les fonctions syntaxiques de ceux-ci. 


\section{LES REMARQUES SUR LA BIBLIOGRAPHIE DU SLOGAN}

La bibliographie concernant le slogan analysé du point de vue de la pragmatique, est très riche aussi bien en français qu'en polonais ce qui prouve l'importance de cette perspective d'envisager la forme linguistique en question. On y trouve aussi bien des articles focalisés sur des problèmes particuliers (Grunig 1998, Dahlet, Dybalska 1998/99, Lisowska-Magdziarz 1998, Szczęsna 2003) que des ouvrages plus vastes (p. ex. Verdier 1982, Charaudeau 1992, Everaert-Desmedt 1984, Skowronek 1993, Bralczyk 2004). C'est un sujet choisi pour les thèses ou mémoires de maîtrise et de licence (Burbea 2006; Brunner 2003, Ferreira, Krzanowska 2008).

Dans la bibliographie française, on précise, entre autres, le but du discours publicitaire, c'est-à-dire «faire faire » (en l'occurrence "faire acheter ») par le biais d'un "faire savoir» (en l'occurrence "faire croire aux qualités supérieures d'un produit), c'est-à-dire de modifier la situation des interlocuteurs à l'égard du produit en question, dès lors qu'ils acceptent d'en effectuer l'achat) (Dahlet: 343). Le même auteur souligne l'appartenance du slogan aux actes indirects en traitant la publicité comme macro-acte de langage («faire acheter») et attire l'attention sur son statut d'une communication-fiction (344-346). Plusieurs slogans sont traités comme énonciations polyphoniques (Barthes 1993). On s'intéresse aussi à la fonction du vous ambigu (Thomas, Van de Ponseele) et à l'ambiguïté du slogan en général (Brunner 2003). On y retrouve aussi l'analyse du caractère du message, celui-ci faisant entendre sans dire ce par quoi on rapproche certains slogans (en changeant l'optique en celle de la rhétorique) des figures de réticence énonciative, mais aussi de l'allusion (Bonhomme 2006: 146-151), etc.

Les auteurs polonais reprennent l'idée d'un macro-acte publicitaire, en précisant les micro-actes accomplis par les slogans polonais dans leur aspect illocutoire et perlocutoire (Skowronek 1993). On souligne la prédominance des actes indirects (Bralczyk 2004). K. Skowronek s'occupe aussi des conditions de félicité, du classement des effets et des intentions de l'émetteur des actes publicitaires. Comme dans la bibliographie française, on analyse la fonction de vous et de tu exprimant le récepteur du message et on attire l'attention sur un moi polyphonique (Dybalska 1998/99). Bralczyk rend compte des stratégies de manipulation en se servant des présuppositions et des implications (1996). Les ééments pragmatiques précités restent en relation étroite avec la publicité subliminale traitée par une riche bibliographie polonaise (Bartkowska-Nowak 1999, Lisowska-Magdziarz 1998, Ohme 2002, Falkowski 2004). C'est grâce aux stratégies de la persuasion subliminale, telles qu'un effet d'exposition pure, le fait de cacher une dépendance entre le stimulus et la réaction, la structuration rétroactive de la mémoire (backward framing) qu'on comprend le fonctionnement des moyens linguistiques relevant de la pragmatique. On ne peut pas non plus passer sous silence le problème de la différence entre la pragmatique publicitaire et la pragmatique de l'humour, abordé par et Szczęsna (2003) qui s'appuie entre autres sur les recherches de Ziomek (1980) consacrées à l'humour. 
On voit que la plupart des questions et des optiques dans lesquelles on analyse le slogan du point de vue de la pragmatique sont les mêmes dans les bibliographies française et polonaise. L'analyse pragmatique des slogans humoristiques semble une particularité polonaise. Dans le cas de certains problèmes, on constate que là où les linguistes français tracent des perspectives de recherches (le classement des micro-actes) (Dahlet), les linguistes polonais les mettent en œuvre (Skowronek 1993).

Le problème des fonctions du langage dans l'optique jakobsonienne, rapporté aux slogans, a été traité explicitement par Reboul (1979). L'auteur décrit la fonction expressive comme originaire pour ces phrases autonymes. La fonction incitative est caractérisée par rapport à celle de la consigne et du mot d'ordre. Dans la description de la fonction référentielle, Reboul souligne le caractère sommaire qui rend le slogan invérifiable et indiscutable. Le pouvoir phatique du slogan ne vient pas, d'après le même auteur, de ce qu'il est répété mais de ce qu'il est répétable. En analysant la fonction métalinguistique, l'auteur constate que les slogans enrichissent la langue. Ces phrases bloquent cette fonction dans la mesure où elles sont sans réplique. $\mathrm{Si}$ elles sont ouvertes, c'est qu'elles ne sont pas réussies. Selon Reboul, la valeur poétique s'exprimant par diverses figures stylistiques est nécessaire pour un bon slogan et le rend intraduisible.

\section{QUELQUES TRAITS DÉFINITOIRES DU SLOGAN ET UNE REMARQUE HISTORIQUE}

Le slogan est une forme autonyme, en général brève, apparentée au proverbe et en tant que telle, il se définit négativement par rapport à ce dernier. Le slogan diffère de celui-ci par le fait d'être non-anonyme. Arnaud remarque judicieusement que «même si son auteur individuel n'est pas connu, il est perçu comme émanant d'un groupe précis, à des fins publicitaires ou bien didactiques », p. ex. Un train peut en cacher un autre (un slogan de la SNCF) ou Quand les parents boivent, les enfants trinquent (destiné à la lutte contre l'alcoolisme) (Arnaud 1991: 11). Par conséquent, la plupart des slogans ne sont pas des dénominations (ne sont pas des signes-phrases, des unités codées ou préconstruites renvoyant à une entité générale, comme les proverbes, donc ils ne font pas partie du code commun et ne figurent pas dans les dictionnaires) n'ayant qu'une existence éphémère et une provenance précise. Un grand nombre d'entre eux, en se référant à un produit particulier ne sont pas des phrases génériques [qui rendent compte d' «un certain état de choses général, habituel ou constant» (Kuroda 1973: 88) «en exprimant des relations devenues indépendantes en quelque sorte des situations particulières, un état de choses ou situation potentiel et non réel » (Kleiber 1994: 217)]. Pourtant dès que les slogans deviennent dénominations (relèvent de l'usage commun) et phrases génériques, ce qui a lieu quand ils se font anonymes et servent de moule à d'autres phrases, ils acquièrent un statut de proverbe (Lipińska 2004: 10-13). 
L'étymologie du slogan est gaélique et reste en rapport avec le cri de guerre d'un clan écossais en datant de 1513. Le message de type publicitaire apparaît vers les années 1922 (Ferreira: 361).

\section{LES FONCTIONS DU LANGAGE}

\subsection{LES FONCTIONS : COMMUNICATIVE ET CONATIVE}

La langue des slogans a des fonctions diverses. A part la fonction communicative découlant de la pertinence du message publicitaire, la fonction injonctive est sans doute centrale et définitoire de ces formes autonymes et toutes les autres fonctions y sont subordonnées. Le slogan, de par sa nature, persuade d' acheter ou de profiter de services, ce qui ne se reflète qu'assez rarement dans la forme linguistique appropriée. Les phrases impératives [p. ex. Laissez-nous vous surprendre (Skôda); Mâchez, respirez (Air waves)] ne concernent que 6\% du corpus et sont presque aussi fréquentes que les phrases interrogatives $[8 \%, \mathrm{p}$. ex. Elle a quelque chose en plus, et si c'était Gemey? (Gemey); A qui le tour? (Loto)] ou exclamatives [8\%, p. ex. C'est de la dynamite! (Ovomaltine); Nartaaaaaaaa! (Narta)]. Non seulement la forme linguistique ne reflète pas le caractère conatif du message mais encore, parfois, celui-ci doit être interprété à rebours, p. ex. Le parfum défendu (Eden de Cacharel). L'analyse pragmatique du corpus (d'une centaine de slogans publicitaires français) a démontré que l'injonction reste cachée dans la plupart des phrases et que les actes directs sont minoritaires par rapport aux actes indirects parmi lesquels nous trouvons aussi bien des exemples de dérivations allusives [les trois quarts des énoncés de notre corpus, $\mathrm{p}$. ex. et surtout la valorisation du produit qui remplace l'injonction : C'est tout ce que j'aime (Mc Donald); On ne peut rêver mieux (Citroën)] que de tropes illocutoires (20\% du corpus), dans lesquels la manipulation dont l'objet est le futur client, est beaucoup plus poussée, p. ex. au moyen de questions rhétoriques qui cachent presque toujours une évaluation positive du produit: Peut-on envisager un repas sans Badoit? (Badoit); Que ferais -je sans toi ? (Pavé d'Affinois); Qui peut résister à sa fraîcheur? (Coca-Cola Light). Par conséquent, les slogans apparaissent comme des messages, dans la plupart des cas détournés de leurs sens directs mais non totalement. Ils sont ingénieux mais plutôt évidents et lisibles et en même temps non banals, non lexicalisés, toujours actifs dans leurs détours persuasifs (Lipińska à paraître). Mentionnons encore deux phénomènes assez caractéristiques de ces phrases. D'abord, l'exposition simple du nom de marque (Narta), cette première remplaçant l'injonction Achetez...: Nartaaaaaaa! Ensuite, tous ces cas où le but de l'injonction, c'est-à-dire le fait d'avoir acheté un produit, reste présupposé. Un tel message peut être perçu comme 
incantation de la réalité, p. ex. Il est odieux, mais c'est divin (yaourt Charles Gervais), On sent tout de suite la différence (Frial), Les brûlures passent et la vie continue (Rennie), Pardon, mais c'est trop bon (fromage Chaussée Aux Moines).

\subsection{LA FONCTION RÉFÉRENTIELLE}

Le caractère manipulateur des slogans qui nous imposent de gré ou de force la marque et le type de produit à acheter, s'exprime par la nature effacée, cachée de la fonction référentielle de ces phrases. Dans la plupart des cas, le référent n'est saisissable que par l'implicature, par les données venant du contexte : l'image ou le son accompagnant le message publicitaire, p. ex. Le tigre est en toi (Frosties de Kellogg's); Made in qualité - Solutions pour une vie active (Nissan); Quoi de plus naturel ? (Weight Watchers). Le grand rôle de la déixis est surtout visible dans les phrases à embrayeurs renvoyant au produit de la marque en question : Qui peut résister à sa fraîcheur? (Coca-Cola Light); Ça désaltère et c'est déjà pas mal (Sprite); Ce n'est que pour les enfants (Petit Ecolier). Plus rares sont les cas où l'on sous-entend soit le type, soit la marque du produit : Avec Sabena, vous y seriez déjà (Sabena); Chipster, fallait pas l'ilnJventer (Chipster Belin); Abusez-en, c'est un yaourt (Crème de Yaourt); Biscuits pour adultes (Pim's). Une surdéfinition référentielle est exceptionnelle : Danger, c'est fruité - Vous abusez trop du thé parfumé Eléphant (Eléphant); Une baguette Baguépi oui oui oui oui (Baguépi). Nous avons aussi affaire à une référence délibérément entravée, dans les cas du référent défini $a$ contrario: A ne pas confondre avec une voiture (Renault Scenic).

\subsection{LES FONCTIONS : POÉTIQUE ET MÉTALINGUISTIQUE}

La fonction poétique, centrée sur les traits caractéristiques du message, n'est pas définitoire des slogans mais reste propre à ces phrases souvent originales du point de vue des procédés rhétoriques ou des particularités de la nature pragmatique ou bien, beaucoup plus rarement, morphologiques.

En adoptant le classement des figures du discours de Fontanier $(1977<1821>)$, nous pouvons constater que les procédés stylistiques qui prévalent sont des figures d'élocution, typiques de la langue parlée. Nous y trouvons aussi bien les figures d'élocution par extension ou par ornement qui «étendent, embellissent, ou même caractérisent une idée principale» (Fontanier $1977<1821>$ : 323) que les figures d'élocution par consonance qui visent «à frapper également l'oreille et l'esprit » (Fontanier $1977<1821>$ : 323). Parmi les premières, on cite les épithètes et les répétitions qui vont souvent de pair: La qualité professionnelle - La qualité professionnelle pour vous (Schwarzkopf); Un goût dément déconseillé aux adultes Absolument déconseillé aux adultes (Golden Grahams de Nestlé). Les secondes 
englobent surtout les rimes [Une baguette Baguépi oui, oui, oui, oui (Baguépi); Chambourcy, oh oui! (Chambourcy)], les paronomases, les anaphores [Une cuillère pour l'envie, une cuillère pour la vie (Liebig)], les dérivations [Vous êtes vivant, alors vivez (Cœur de Lion)] et les antanaclases [Petit à petit, on devient moins petit (Danone à boire)]. Les tropes en un seul mot ou proprement dits (métaphores, métonymies) et improprement dits ou en plusieurs mots contribuent au caractère indirect et imagé du message. Voilà quelques exemples de métaphores ou de tropes par ressemblance: Le tigre est en toi (Frosties de Kellogg's) ; C'est de la dynamite! (Ovomaltine) et de métonymie ou de trope par correspondance : Quand on a un cerveau, on a une Fiat Punto (Fiat Punto). Parmi les tropes improprement dits, on observe des figures d'expression par réflexion telles que la réticence qui consiste à laisser entendre ce que l'on tait : Tous les prétextes sont bons (Célébrations) ; La vie est trop courte (Banque Directe); le paradoxe : Deviens ce que tu es (Lacoste), Il est odieux mais c'est divin (Charles Gervais) et l'hyperbole : Plutôt mourir que d'en perdre une miette (Daunat); Un goût dément déconseillé aux adultes - Absolument déconseillé aux adultes (Golden Grahams de Nestlé). Le dernier slogan est en même temps un exemple d'antiphrase, figure d'expression par opposition. Contrairement aux tropes proprement dits, les moyens rhétoriques ci-dessus ne présentent pas une simple idée mais une pensée plus ou moins déguisée ou détournée. On ne les emploie pas par nécessité mais par choix, par artifice (Fontanier 1977 $<1821>$ : 109).

Au chapitre des figures de construction, telles que le rythme et le pléonasme, le parallélisme syntaxique et l'ellipse, les deux premières appartiennent selon Fontanier aux figures par exubérance, $\mathrm{p}$. ex. Une cuillère pour l'envie, une cuillère pour la vie (Liebig) - 2x6 pieds ; Danger, c'est fruité - Vous abusez trop du thé parfumé Eléphant (Eléphant); C'est ceux qui en parlent le moins qui en mangent le plus A consommer de préférence avant les autres (Mc Cain); Quand on a un cerveau, on a une Fiat Punto (Fiat Punto). L'ellipse du verbe est une figure de construction par sous-entente: A qui le tour? (Loto); Un Ricard sinon rien (Ricard). Dans les cas précités, c'est la structure de la phrase qui est arrangée d'une manière spécifique. Nous avons affaire ou à une construction abondante et expansive ou, au contraire, réduite et laconique (Fontanier $1977<1821>$ : 283).

Les figures de style, telles que les métaplasmes (aphérèse, syncope) et la question rhétorique donnent à la pensée plus de relief, en modifiant la forme des mots ou en employant l'emphase: Y'a qu'ta mère pour croire que c'est de l'eau (P'tit Vitel); Y'a pas d'erreur, c'est lessieur (Lessieur); Que ferails (?)]-je sans toi ? (Pavé d'Affinois); Qui peut te battre? (Croustibat de Findus).

L'originalité du message, expliquée pragmatiquement, se rapporte à des jeux de mots concernant l'énoncé sous-entendu, au contraste des implications axiologiques, à la violation des implicatures conventionnelles et au détournement des énoncés échoiques. 
Dans Assurément humain (AGF) et Merci vous aussi (Zappétit), ce qui pique la curiosité, c'est l'association aux noms de marques implicites (le groupe d'assurances et le nom évoquant une phrase idiomatique plaisante Bon zappétit). Nous notons le contraste des implications axiologiques dans C'est bon la honte (Senoble) et Il est odieux mais c'est divin (Charles Gervais). La violation de l'implicature conventionnelle concerne soit la connotation péjorative de certains lexèmes soit la valeur contrastive des sens introduite par le connecteur mais. Dans Abusez-en, c'est un yaourt (Crème de Yaourt), le verbe abusez, ordinairement associé à une action jugée négativement, ordonne ici de persévérer dans cette action dans le but de souligner que le yaourt est délicieux et très bon pour la santé. Il en va de même pour l'énoncé C'est bon la honte (yaourt Senoble) dans lequel le sentiment en question, évalué dans la plupart des cas négativement, est jugé positivement pour la même raison que dans le slogan précédent.

Dans les énoncés C'est frais mais c'est pas grave (chewing-gum Kiss Cool) et Des pâtes, des pâtes, oui mais des Panzani - Laissez parler les Panzani (Panzani), au moyen de la conjonction de coordination mais, on introduit le contraste là où il n'y en a pas. De plus, dans la première phrase, c'est pas grave implique un fait ou une caractéristique négative, tandis que la fraîcheur du chewing-gum est une qualité recherchée. Nous avons donc affaire précisément à la violation de deux implicatures : celle du connecteur et celle de l'expression c'est pas grave. Dans le deuxième slogan, mais implique que des Panzani ne sont pas des pâtes, ce qui est faux. En suivant le principe de coopération, il faut interpréter cet énoncé d'une façon axiologique, imposée par la forme de la phrase citée : c'est plus que des pâtes ordinaires, tellement elles sont délicieuses (Lipińska à paraître).

Le détournement d'un énoncé échoïque concerne soit des syntagmes figés [Made in qualité - Solutions pour une vie active (Nissan); Le parfum défendu (Eden de Cacharel], soit des phrases idiomatiques [Il faut secouer, sinon la pulpe, elle reste en bas - En cas de soif, appelez Orangina (Orangina); Mâchez, respirez (Air waves) : Inspirez profondément, expirez lentement! ; C'est ceux qui en parlent le moins qui en mangent le plus - le slogan des frites Mac Cain, dérivé de la phrase C'est ceux qui en parlent le plus qui en font le moins, utilisée en parlant de ceux qui se vantent inconsidérément, en qualité et/ou en quantité, de leurs exploits amoureux, soit des proverbes [Et vos idées ont du génie - L'appétit vient en Maggi (Maggi) : L'appétit vient en mangeant; Qui sème les fleurs, récolte la tendresse (Interflora): Qui sème le vent récolte la tempête]. L'originalité des phrases précitées qui renvoient en mêmes temps aux autres phrases envisagées comme eléments du code, permet de situer ces exemples non seulement parmi les preuves de la fonction poétique mais aussi confirme la présence de la fonction métalinguistique dans certains slogans. En adoptant ce point de vue, on ne peut pas être d'accord avec l'opinion de Reboul (1979) sur le blocage de la fonction métalinguistique par les slogans. 
Il y a enfin des slogans qui renvoient aux slogans antérieurs de la même marque, p. ex. Nartaaaaaaaaaaaa! (Narta) dont la forme du message peut attirer aussi l'attention du récepteur, à savoir un allongement artificiel d'un phonème, qu'on retrouve dans les énoncés exclamatifs, très expressifs. La phrase renvoie au slogan des années 1980 C'est fou le frais que tu me fais Nartaaaa! On pourrait faire la même observation au sujet de la marque Orangina, qui, d'une manière ou d'une autre, invite ses clients à secouer la bouteille depuis les années 1930. Le slogan d'origine, «Il faut secouer la bouteille d'Orangina pour bien mélanger la pulpe d'orange », est passé par une série d'états ("secouez et buvez»; «secouez-moi, secouez-moi, Orangina ») qui permet à la marque de faire allusion à elle-même.

Le message du slogan publicitaire est donc original dans sa forme et dans son sens plus ou moins explicite. Il pique la curiosité par toutes sortes de procédés rhétoriques et d'artifices pragmatiques. Sa construction découle d'une réflexion profonde sur l'utilisation de moyens linguistiques variés, visant l'effet de surprise, de comique, d'étonnement, lesquels sont subordonnés à la force injonctive et à l'impact publicitaire.

\subsection{LA FONCTION PHATIQUE}

Capter et retenir l'attention du récepteur est donc un objectif crucial pour les publicitaires, voilà pourquoi on retrouve aisément dans les slogans les indices de la fonction phatique de ces phrases. Pour entrer en contact, une question est un procédé très effectif. En sus des questions rhétoriques, nous observons les cas d'interrogation directe qui a souvent la forme d'une proposition aimable: Si on en parlait? (Société Générale); Est-ce que vous le ressentez? (Reebok); It's a bit strong !/C'est fort! Et vous? (Fisherman's Friend). Le rôle des phrases classées par Grevisse comme affectives: exclamatives et impératives, est semblable: Nartaaaaaaaaaaaa! (Narta); Abusez-en, c'est un yaourt! (Crème de Yaourt). Le monologue intérieur avec lequel le récepteur du slogan peut s'identifier et le renforcement des émotions positives, p. ex. par la valorisation du client, ont une grande force persuasive mais aussi permettent d'entrer en contact et de fixer l'attention : Je suis pas jolie, je suis pire (Kenzo); Parce que je le vaux bien (L'Oréal). En général, la présentation des émotions et des jugements de valeur positifs possède une fonction compensatrice et par conséquent phatique : Pardon, mais c'est trop bon! (Chaussée aux Moines).

\subsection{LA FONCTION EXPRESSIVE}

La fonction expressive n'est pas non plus définitoire des slogans mais elle reste représentative d'un grand nombre de ces phrases. La valeur expressive est prise en charge par des moyens linguistiques variés : des propriétés syntaxiques des énon- 
cés, des indices d'appartenance aux registres marqués, des figures de style, des émotions contenues dans les messages et enfin des éléments évaluatifs.

Il faut y mentionner des phrases affectives et la mise en relief dans les énoncés typiques de la langue parlée et familière. Aussi, toutes les figures rhétoriques précitées contribuent-elles à la valeur expressive des énoncés. Les traces des registres marqués tout en relevant des connotations à caractère syntaxique, morphologique, lexical, phraséologique ou pragmatique, nous apportent des informations sur l'émetteur du message (Lipińska à paraître).

Les émotions exprimées sont positives (satisfaction, gratitude, liaison forte, dévouement), p. ex. C'est bon de reboire du lait (Matin Léger de Lactel), Que feraisje sans toi ? (Pavé d'Affinois), Quotidiennement vôtres (CCP et Chèques Postaux) ainsi que les évaluations véhiculées fréquemment par la partie dénotative ou connotative des lexèmes ou bien par une implication du message. Voilà quelques exemples de dénotations mélioratives des lexèmes: La qualité professionnelle, la qualité professionnelle pour vous (Schwarzkopf); Quand c'est bon, c'est Bonduelle! (Bonduelle); Made in qualité - Solutions pour une vie active (Nissan); Le plaisir est complet (Wasa) et des mots marqués par une connotation méliorative: De la vie en veux-tu, en voilà (Viva de Candia); Qui peut résister à sa fraîcheur? (CocaCola Light); Ravi que ça vous plaise (Pains Jacquet). Un autre moyen sémantique de faire augmenter la valeur expressive du message est un contraste d'évaluation ou une axiologie à rebours, p. ex. C'est bon la honte (Senoble) et Il est odieux mais c'est divin (Charles Gervais); Qui peut dominer une méchante envie de Côte d'Or? (Côte d'Or). Les informations impliquées soulignent une bonne qualité des services ou celle du produit : Nous ne sommes pas populaires sans raison - on suggère que le nom et en même temps la popularité de la Banque Populaire viennent de la grande qualité de ses services ; On n'y résiste pas! parce que les biscuits Quadro sont très bons.

Le caractère plaisant, léger ou humoristique des slogans est aussi à l'origine de leur force expressive : La petite faiblesse qui vous perdra (Mikado); Une baguette Baguépi oui oui oui oui (Baguépi).

Ce n'est que très rarement que nous avons affaire à une évaluation négative : Je ne suis pas jolie, je suis pire (Kenzo). Cette exception ne fait que confirmer la règle découverte par plusieurs linguistes, à savoir que la publicité évite les eléments dépréciatifs, négatifs y compris dans le sens de la négation du verbe et favorise des lexèmes mélioratifs, euphémiques ainsi que la forme affirmative (Dybalska 1998/99: 87-88).

\section{LES DÉICTIQUES}

La déixis, c'est-à-dire le recours à la situation de communication, joue un rôle considérable dans les slogans, ce qui est lié au statut de ces derniers, c'est-à-dire qu'ils constituent des phrases qui ne sont ni des phrases génériques ni des dénomi- 
nations. Le degré d'actualisation de ces énoncés est assez élevé ; dans notre corpus, on a relevé $60 \%$ des phrases avec des embrayeurs qu'on trouve parfois plusieurs fois dans un énoncé, p. ex. Parce que je le vaux bien (L'Oréal); It's a bit strong ! / C'est fort! Et vous? (Fisherman's Friend) ; Vous êtes différent, nous aussi (Banque Covefi).

Les déictiques s'avèrent bien diversifiés : à partir des pronoms personnels, démonstratifs, possessifs, des adjectifs possessifs jusqu'aux adverbes de temps et aux pronoms indéfinis.

Les pronoms personnels sujet sont les plus nombreux ( $40 \%$ des slogans). Le plus fréquemment, on emploie un vous ambigu représentant le/s récepteur/s client/s potentiel/s- personnage exemplaire qui a souvent acheté et utilisé le produit dont on fait la publicité et qui en est très content, p. ex. Danger, c'est fruité - Vous abusez trop du thé parfumé Eléphant (Eléphant); Ravi que ça vous plaise (Pains Jacquet). Nous remplace presque toujours l'émetteur, celui qui a créé le produit ou offre un service, p. ex. bancaire : Laissez-nous vous surprendre (Skôda); Nous ne sommes pas populaires sans raison (Banque Populaire). Il est parfois accompagné d'un récepteur virtuel ou bien, exceptionnellement nous se rapporte aux personnages d'un film : Forever sports - La victoire est en nous - On y va pour gagner (Adidas); Nous n'avons pas les mêmes valeurs (Rillettes Bordeau Chesnel).

Le rôle de on est dans certains slogans semblable car ce pronom représente l'émetteur-producteur accompagné du récepteur-client qui utilisent le produit ou profitent de certains services: On n'y résiste pas! (biscuits Quadro) ; On trouve tout ? la Samaritaine (magasin La Samaritaine). Mais il y a aussi des cas où on se réfere à un groupe spécifique de clients-destinataires, tels que les enfants : Petit à petit, on devient moins petit (yaourt Danone à Boire).

$J e$ a des fonctions diverses : soit, le plus souvent, il représente un personnage exemplaire qui a acheté et utilisé le produit, soit le destinataire du slogan, un client virtuel: Que ferai-je sans toi? (fromage Pavé d'Affinois); Parce que je le vaux bien (L'Oréal) ; C'est tout ce que j'aime (Mc Donald) ou bien Je ne suis pas jolie, je suis pire (cosmétiques Kenzo). Tu se réfère au destinataire, le futur client : De la vie en veux-tu, en voilà (lait Viva De Candia); Deviens ce que tu es (vêtements Lacoste). Toi se rapporte soit au produit personnalisé : Que ferai-je sans toi ? (fromage Pavé d'Affinois), soit au destinataire-enfant: A toi d'imaginer (Lego); Le tigre est en toi (Frosties de Kellogg's). Les pronoms sujet de la troisième personne représentent, soit le produit, soit, moins fréquemment, son utilisateur : Il est odieux, mais c'est divin (yaourt Charles Gervais); S'ils sont bons, c'est qu'ils sont faits à sa façon (Les Secrets de Pauline) ; Elle a quelque chose en plus, et si c'était Gemey? (Gemey).

Nous n'avons relevé qu'un exemple de pronom personnel atone en fonction de COD : te, lequel remplace le personnage de bande dessinée symbolisant le produit : Qui peut te battre? (Croustibat De Findus). La même fonction du pronom le est associée aux avantages du produit d'une marque précise, ces derniers étant sous- 
entendus: Est-ce que vous le ressentez? (Reebok); Parce que 'je le vaux bien (L'Oréal).

$Y$ et $e n$, en fonction de COI, reprennent aussi les éléments sous-entendus principaux, c'est-à-dire le produit d'une marque précise : On n'y résiste pas ! (biscuits Quadro), et d'une manière cataphorique Abusez-en, c'est un yaourt (Crème de Yaourt). $Y$ en fonction de $\mathrm{CC}$ de lieu représente l'endroit où le récepteur se trouve ou veut aller virtuellement : Avec Sabena, vous y seriez déjà (Sabena), ou bien, par anaphore, l'élément précédent de l'énoncé : Forever sports - La victoire est en nous - On y va pour gagner (Adidas) - y remplaçant à la compétition. En dans des fonctions différentes (CC de propos, COD, complément déterminatif du nom) se rapporte aussi au produit d'une marque précise ou au service proposé, qui reste sous-entendu : C'est ceux qui en parlent le moins qui en mangent le plus - A consommer de préférence avant les autres (Mc Cain); Si on en parlait? (Société Générale); Plutôt mourir que d'en perdre une miette (Daunat). Le même pronom avec le référent précité peut être anaphorique du COD : De la vie en veux-tu, en voilà (lait Viva De Candia).

Les pronoms démonstratifs déictiques apparaissent dans $20 \%$ des slogans. $C^{\prime}$ (le plus fréquent), ça, ce s'emploient tous en fonction de sujet et, à deux exceptions près, se réfèrent au produit d'une marque précise, les deux étant sous-entendus : C'est fait par qui ? Par Picard (Surgelés Picard); C'est moche mais ça marche (Eau précieuse); Ce n'est que pour les enfants (biscuits Petit Ecolier). Ce n'est que très rarement qu'ils ont un caractère anaphorique : ll est odieux, mais c'est divin (yaourt Charles Gervais); L'ingrédient le plus actif, c'est vous (dentifrice Signal).

Les adjectifs et le pronom possessifs employés en fonction d'embrayeurs représentent $6 \%$ du corpus. Dans la plupart des cas, ils déterminent le nom en fonction de sujet et se rapportent aux clients potentiels ou exemplaires : C'est mon faible (Foie Gras Montfort); Et vos idées ont du génie - L'appétit vient en Maggi (Maggi); N'attendez pas que votre voiture vous le demande (Speedy). Ce n'est qu'exceptionnellement qu'ils se réfèrent au prestataire du service ou à la marque du produit: Quotidiennement vôtres (CCP et Chèques Postaux); S'ils sont si bons, c'est qu'ils sont faits à sa façon (Les secrets de Pauline).

Les autres déictiques ont le statut d'hapax. On y trouve l'adverbe de temps déjà [Avec Sabena, vous y seriez déjà (Sabena)] et le pronom indéfini un tel à l'intérieur d'un syntagme en fonction de COI se rapportant aux avantages du produit [Qui peut résister à un tel luxe ? (Lanvin)].

\section{CONCLUSION}

Pour résumer notre description des fonctions du langage dans les slogans, nous pouvons constater qu'il faut traiter les fonctions communicative, conative et phatique comme définitoires de ce type de phrases, la fonction incitative étant souvent délibérément cachée. Les fonctions poétique et expressive sont très importantes 
mais non nécessaires, tandis que la fonction référentielle reste effacée dans un grand nombre de cas. La fonction métalinguistique joue un rôle moindre mais on ne peut pas l'exclure.

L'analyse de la déixis montre que c'est le type de message qui impose à l'énoncé les référents principaux des déictiques ainsi que les fonctions syntaxiques les plus courantes de ceux-ci. Nous trouvons ici, surtout en fonction de sujet le produit, sa marque, le producteur ou le prestataire d'un service, le client potentiel ou le personnage exemplaire qui a utilisé un produit et en est très content. La déixis du slogan publicitaire semble focalisée sur les éléments principaux du point de vue de la vente du produit. travail.

Je remercie Olivier Guionaud de ses remarques pertinentes qui ont enrichi ce

\section{BIBLIOGRAPHIE}

Arnaud P.J.L. (1991), « Réflexions sur le proverbe », Cahiers de lexicologie, 59-62, p. 6-27.

Barthes R. (1993), «L'empire des signes », in : E. Marty (ed.), Barthes. Oeuvres complètes. 19661973, p. 741-831.

Bartkowska-Nowak D. (1999), «Niewidoczne sznurki », Charaktery, ${ }^{0} 7$ (30), p. 19-20.

Bonhomme M. (2006), Le discours métonymique, collection «Sciences pour la communication», Berne : Peter Lang.

Bralczyk J. (2004), Język na sprzedaż, Gdańsk : Gdańskie Wydawnictwo Psychologiczne.

Brunner C. (2003), L'ambiguïté dans le slogan publicitaire, dir. J.-M. Adam.

Charaudeau P. (1992), Grammaire du sens et de l'expression, Paris : Hachette.

Dybalska R. (1998/99), « Czy białe może być czarne ? O reklamowych mechanizmach językowej manipulacji », Języki obce w szkole, cahier 6, p. 84-91.

Everaert-Desmedt N. (1984), La communication publicitaire: êtude semio-pragmatique, Louvain-laNeuve : Cabay.

Falkowski A. (2004), « Mieszanie w pamięci », Charaktery, nº 2, p. 34-35.

Fontanier $P$. $(1977<1821>)$, Les figures du discours, Paris : Flammarion.

Grunig B.-N. (1998), Les mots de la publicité: l'architecture du slogan, Paris : Presses du CNRS. D. L. Kerbrat-Orecchii C. (1986), L'implicite, Paris : A. Colin.

Kleiber G. (1994), Nominales, Paris : A. Colin.

Kuroda S .Y. (1973), « Le jugement catégorique et le jugement thétique. Exemples tirés de la syntaxe japonaise ", Langages, 29, p. 81-110.

Lipińska M. (2004), L'équivalence des proverbes polonais et des proverbes français, tódź : Wydawnictwo Uniwersytetu Łódzkiego.

Lipińska M., «L’analyse pragmatique des slogans français », à paraître.

Lipińska M., «Les registres de langue dans les slogans publicitaires français », à paraître.

Lisowska-Magdziarz M. (2007), Analiza tekstu w dyskursie medialnym. Przewodnik dla studentów, Kraków : Uniwersytet Jagielloński.

Ohme R. K. (2002), « Nowe spojrzenie na Reklamę », Charaktery, $n^{\circ} 10$, p. 37-39. 
Reboul O. (1979), «Le slogan et les fonctions du langage ", Français dans le monde, n 143, p. 21-26.

Skowronek K. (1993), Reklama : studium pragmalingwistyczne, Kraków : Polska Akademia Nauk. Instytut Języka Polskiego.

Szczęsna E. (2003), « Humor w reklamie », Przeglad Humanistyczny, n 3, p. 5-12.

Verdier H. (1982), La publicité, Paris : PUF.

Ziomek J. (1980), « Komizm - spójność teorii i teoria spójności », in : Powinowactwa literatury, Warszawa : PWN.

\section{SITES D'INTERNET}

Brunner C., «L'ambiguïté dans le slogan publicitaire », in : www.unil.ch/fra/pagel6675.html - 46k

Burbea G., «Le slogan publicitaire, stratêgies pragmatiques », in : www.u- picardie.fr/labo/curapp/CV/ CVRichard2.pdf

Cubillo Ferreira C. D. «Le slogan publicitaire : une lecture des notions pour la vente des produits et des services automobiles », in : www.dialnet.unirioja.es/sevlet/fichero.articulo ?codigo $=1011578 \&$ orden $=74688$

Dahlet $\mathrm{P}$., «Un langage efficace : l'illocution indirecte. Contribution à une représentation pragmatique du discours publicitairte ", in : www.ler.letras.up.pt/uploads/ficheiros/artigo6121.pdf

Krzanowska A., « Semantyczno-pragmatyczna analiza języka sloganu reklamowego (na materiale języka polskiego i rosyjskiego », in : www.kul.lublin.pl/art_3429.html

Thomas F., Van de Ponseele O., in : www.echoscommunication.org/html/project_01_g.html 